\title{
Prevalência e fatores associados a comportamentos de risco à saúde em universitários no norte de Minas Gerais
}

\author{
Prevalence and factors associated with health risk \\ behaviours in university students on the north of Minas Gerais
}

\author{
Celina Aparecida Gonçalves Lima ${ }^{1}$, \\ Maria de Fátima de Matos Maia², Tatiana Almeida de Magalhães ${ }^{3}$, \\ Liliane Marta Mendes de Oliveira ${ }^{3}$, Vivianne Margareth Chaves Pereira Reis ${ }^{4}$, \\ Maria Fernanda Santos Figueiredo Brito ${ }^{5}$, Lucineia de Pinho ${ }^{6}$, \\ Marise Fagundes Silveira ${ }^{1}$
}

\begin{abstract}
Resumo
Objetivo: Estimar a prevalência de comportamentos de risco à saúde (CRS) e identificar os fatores associados. Métodos: Estudo transversal com amostra aleatória de 902 acadêmicos de uma universidade pública no norte de Minas Gerais, Brasil. As variáveis analisadas foram: características sociodemográficas, acadêmicas e comportamentos de risco. Utilizou-se a Razão de Prevalência bruta e ajustada, estimadas pelo modelo de regressão de Poisson. Resultados: Os CRS mais prevalentes foram: baixo consumo de frutas e verduras $(98,1 \%)$, não realização de exercício aeróbio $(71,2 \%)$ e uso irregular de preservativo $(63,1 \%)$. As associações significativas foram: gênero masculino com consumo abusivo de bebida alcoólica $(R P=1,53)$, drogas ilícitas $(R P=2,41)$ e envolvimento em brigas ( $R P=2,12)$; idade até 21 anos com envolvimento em brigas $(R P=2,77)$; estado civil com companheiro com envolvimento em brigas $(R P=3,03)$ e uso irregular de preservativo $(R P=1,59)$; estudantes de outras áreas de graduação com não realização de exercício aeróbio $(R P=1,26)$; classe econômica $A$ ou $B$ com drogas ilícitas $(R P=1,92)$. Conclusão: Observaram-se variações nas prevalências dos CRS, com destaque para baixo consumo de frutas e verduras, e tabagismo. Os CRS mostraram-se positivamente associados ao gênero masculino, idade até 21 anos, classe econômica A ou B, estado civil com companheiro e estudantes de outras áreas de graduação.
\end{abstract}

Palavras-chave: comportamento de risco; universitários; saúde.

\footnotetext{
Abstract

Objectives: To estimate the prevalence of health risk behaviors (HRB) and identify the factors associated with it. Methods: Cross-sectional study with a random sample of 902 students, at a public university on the north of Minas Gerais, Brazil. The variables analyzed were: sociodemographic characteristics, academic and risk behaviors. It was used the crude and adjusted prevalence ratio, estimated by the Poisson model. Results: HRB more prevalents: low consumption of fruits and vegetables (98.1\%), non-performance of

'Departamento de Ciências Exatas, Universidade Estadual de Montes Claros (Unimontes) - Montes Claros (MG), Brasil.

${ }^{2}$ Grupo Integrado de Pesquisa em Psicologia do Esporte, Exercício e Saúde, Saúde Ocupacional e Mídia (GIPESOM), Universidade Estadual de Montes Claros (Unimontes) - Montes Claros (MG), Brasil.

3Programa de Pós-graduação em Ciências da Saúde, Universidade Estadual de Montes Claros (Unimontes) - Montes Claros (MG), Brasil.

${ }^{4}$ Departamento de Educação Física, Faculdades Unidas do Norte de Minas (FUNORTE) - Montes Claros (MG), Brasil.

${ }^{5}$ Departamento de Saúde Mental e Saúde Coletiva, Universidade Estadual de Montes Claros (Unimontes) - Montes Claros (MG), Brasil.

${ }^{6}$ Departamento de Fisiopatologia, Universidade Estadual de Montes Claros (Unimontes) - Montes Claros (MG), Brasil.

Trabalho realizado na Universidade Estadual de Montes Claros (Unimontes) - Montes Claros (MG), Brasil.

Endereço para correspondência: Celina Aparecida Gonçalves Lima - Departamento de Ciências Exatas, Universidade Estadual de Montes Claros (Unimontes), Rua Professor Antônio Sapucaí, 66 - Cristo Rei - CEP: 39402-391 - Montes Claros (MG), Brasil - Email: celina.prof@bol.com.br

Fonte de financiamento: nenhuma.

Conflito de interesses: nada a declarar.
} 
aerobic exercise (71.2\%) and irregular preservative use (63.1\%). The significant associations were: males with abusive consumption of alcohol $(R P=1.53)$, illegal drugs $(R P=2.41)$ and involvement in fights $(R P=2.12)$; age up to 21 years with involvement in fights $(\mathrm{RP}=2.77)$; relashionship status with a partner with involvement in fights $(\mathrm{RP}=3.03)$ and irregular use of preservatives $(\mathrm{RP}=1.59)$; students from other academic areas with non-performance of aerobic exercise $(R P=1.26)$; economic class $A$ or $B$ with illicit drugs $(\mathrm{RP}=1.92)$. Conclusion: There were variations in the prevalence of HRB among university students, with emphasis on low fruit and vegetable consumption and smoking. HRB were positively associated with the male gender, up to 21 years, economic class A or $\mathrm{B}$, marital status with partner and students from other undergraduate areas.

Keywords: risk behavior; college students; health.

\section{INTRODUÇÃO}

Os comportamentos de risco à saúde (CRS) contribuem para as principais causas de morbimortalidade entre adolescentes $\mathrm{e}$ jovens universitários ${ }^{1-3}$. Pesquisas desenvolvidas em diferentes partes do mundo indicaram que os CRS entre os universitários são o sedentarismo, os distúrbios alimentares, os acidentes no trânsito, o uso de tabaco, álcool e outras drogas, o envolvimento em situações de violência e as condutas sexuais de risco ${ }^{1-4}$. Esses CRS podem levar à morbimortalidade por favorecerem $\mathrm{o}$ desenvolvimento de doenças como as sexualmente transmissíveis, gravidez de alto risco, disfunções orgânicas e doenças crônicas não transmissíveis ${ }^{1}$, tais como doenças cardiovasculares, diabetes, hipertensão, aterosclerose, acidente vascular cerebral e câncer ${ }^{5-7}$.

Dentre os vários CRS, destaca-se o consumo abusivo de álcool e outras drogas ${ }^{8,9}$. O estilo de vida atual, com elevados índices de stress, ansiedade, baixa autoestima, pressão social e problemas relacionados à escola, tem contribuído para o aumento do consumo excessivo de álcool e outras drogas entre os jovens. Como consequência, surgem vários problemas sociais, familiares, problemas no trabalho e a perda do rendimento escolar ${ }^{9-11}$.

Observa-se também que a adoção de comportamento sedentário e uma dieta inadequada aumentam o risco de desenvolvimento e agravamento das doenças crônicas degenerativas, além de elevar os gastos econômicos do indivíduo enfermo, da família e da sociedade ${ }^{12}$.

Assim, o monitoramento dos fatores de risco e da prevalência das doenças a eles relacionados é de fundamental importância para a elaboração de políticas de saúde e estratégias de intervenção e ações educativas desenvolvidas nas universidades e em ambientes de trabalho, a fim de prevenir seus agravos ${ }^{9,13}$.

O aumento da população de universitários no Brasil, registrado nas últimas décadas, e sua vulnerabilidade aos CRS têm despertado o interesse de pesquisadores que vêm desenvolvendo estudos epidemiológicos com esse tema ${ }^{14,15}$. Entretanto, os dados são ainda escassos para algumas regiões do país ${ }^{7,15}$, e a maioria dos trabalhos identificados na literatura investigou adolescentes e não universitários ${ }^{5}$. Na região norte de Minas Gerais, foram identificados apenas dois estudos sobre CRS de universitários, ambos relacionados ao consumo de bebida alcóolica ${ }^{16,17}$. Assim, é importante conhecer os CRS desses jovens, pois, ao ingressarem na universidade, adquirem mais liberdade e independência, ficando mais expostos e vulneráveis a comportamentos de risco à saúde ${ }^{18}$.

O padrão de comportamento dos universitários tem sido modificado com adoção de estilos de vida e, visto que eles representam um importante segmento da população jovem, $o$ impacto dessas alterações deveria ser debatido nas universidades ${ }^{15}$. Informações sobre os CRS entre os que frequentam a universidade podem contribuir para o monitoramento de grupos de risco e desenvolvimento de políticas e programas de promoção à sua saúde ${ }^{5,14}$. Sendo assim, o presente trabalho objetivou estimar a prevalência de CRS em uma população de universitários do norte de Minas Gerais, bem como identificar os fatores associados a esses comportamentos.

\section{MÉTODOS}

\section{Sujeitos da pesquisa e delineamento do estudo}

O presente estudo é um recorte do projeto "Comportamentos de risco para a saúde de acadêmicos da Universidade Estadual de Montes Claros - Unimontes", realizado pelo GIPESOM (Grupo Integrado de Pesquisa em Psicologia do Esporte, Exercício e Saúde, Saúde Ocupacional e Mídia), vinculado à instituição. Em uma abordagem transversal, buscou-se amostrar uma população-alvo constituída por 7.868 universitários matriculados nos cursos de graduação presenciais oferecidos pela Unimontes em 2013. A Unimontes é uma instituição pública de ensino superior, localizada na cidade de Montes Claros, MG. Oferecendo cursos nas áreas de saúde, educação, ciências sociais aplicadas, ciências humanas e ciências exatas e tecnológicas, o campus-sede da Unimontes em Montes Claros e seus doze campi (em Almenara, Bocaiúva, Brasília de Minas, Espinosa, Janaúba, Januária, Joaíma, Paracatu, Pirapora, Salinas, São Francisco e Unaí) formam um polo educacional para o qual convergem estudantes de outros municípios da região do norte de Minas Gerais.

\section{Amostragem}

$\mathrm{Na}$ determinação do tamanho da amostra, em função dos múltiplos CRS investigados na pesquisa, considerou-se uma prevalência máxima esperada de 50\%, com nível de confiança de 
95\% e margem de erro de 5\%. Foi também realizada a correção pelo efeito do desenho, adotando-se deffigual a dois e acréscimo de $20 \%$ para taxa de não resposta. Determinou-se, assim, uma amostragem mínima de 960 indivíduos.

Os estudantes foram selecionados por amostragem probabilística por conglomerado em dois estágios. No primeiro estágio, foram selecionados por amostragem aleatória simples 50\% dos cursos de cada área de conhecimento (Ciências Sociais Aplicadas, Ciências Exatas e Tecnológicas, Ciências Humanas e Ciências Biológicas e da Saúde). No segundo estágio, sortearam-se também aleatoriamente $25 \%$ das turmas de cada curso previamente selecionado. Os alunos dessas turmas que estavam presentes no momento da aplicação do questionário foram convidados a participar da pesquisa.

\section{Variáveis do estudo e coleta de dados}

Utilizou-se o questionário Youth Risk Behavior Survey - College $(\text { YRBS-C })^{15}$ para coleta dos dados referentes às características sociodemográficas e CRS dos acadêmicos. A classe econômica foi definida pelo Critério de Classificação Econômica Brasil da Associação Brasileira de Empresas de Pesquisa - ABEP ${ }^{19}$. As características investigadas foram alocadas em três grupos de variáveis: comportamentos de risco, características sociodemográficas e acadêmicas. As variáveis investigadas no estudo e suas respectivas categorias estão descritas no Quadro 1. Antes de responder aos questionários, os estudantes foram informados sobre os objetivos da pesquisa e sobre a preservação do anonimato. Os questionários foram aplicados nas salas de aula por equipe capacitada e sob a supervisão dos pesquisadores responsáveis pelo projeto. Após preenchimento, os estudantes depositaram os questionários em um envelope sem identificação. A coleta de dados ocorreu no período agosto a dezembro de 2013.

\section{Análise dos dados}

As variáveis analisadas foram descritas por frequências absolutas e relativas, com correção pelo efeito do desenho (deff). As prevalências dos CRS foram estimadas pelos seus intervalos de confiança de 95\%. As características sociodemográficas e acadêmicas foram consideradas como variáveis independentes e os CRS como variáveis dependentes (desfechos). A magnitude da associação entre as variáveis dependentes e independentes foi avaliada pela Razão de Prevalência (RP) bruta e ajustada, estimada mediante o modelo de Poisson com variância robusta. Inicialmente foram realizadas análises bivariadas, e as variáveis que apresentaram nível descritivo (valor-p) até 0,20 foram selecionadas para o modelo múltiplo (análise ajustada), com nível de significância de 0,05 . O teste deviance foi utilizado para avaliar a qualidade dos modelos ajustados. Todas as análises estatísticas foram realizadas utilizando-se o programa Predictive Analytics Software (PASW) ${ }^{\circledR}$ versão 19.0 para Windows ${ }^{\circledR}$.

\section{Aspectos éticos}

Este estudo foi aprovado pelo Comitê de Ética em Pesquisa da Universidade Estadual de Montes Claros (Parecer no 30679/2012). Todos os universitários que concordaram em participar do estudo assinaram um Termo de Consentimento Livre e Esclarecido - TCLE.

Quadro 1. Comportamentos de risco e variáveis sociodemográficas e acadêmicas avaliados entre universitários. Norte de Minas Gerais, Brasil, 2013

\begin{tabular}{|c|c|c|}
\hline Variáveis & Medidas (autorreferidas) & Categorias \\
\hline \multicolumn{3}{|l|}{ Comportamentos de risco } \\
\hline \multirow[b]{2}{*}{$\begin{array}{l}\text { Não realização de exercício } \\
\text { aeróbio }\end{array}$} & \multirow{2}{*}{$\begin{array}{l}\text { Frequência semanal de exercício aeróbio, como corridas, } \\
\text { esteiras, bicicletas, ou prática de esportes por pelo menos } 20 \\
\text { minutos por dia, nos últimos } 7 \text { dias anteriores à coleta de } \\
\text { dados }^{20,21} \text {. }\end{array}$} & Sem risco: $\geq 3$ dias \\
\hline & & Com risco $<3$ dias \\
\hline \multirow{2}{*}{$\begin{array}{l}\text { Baixo consumo de frutas e } \\
\text { verduras }\end{array}$} & \multirow{2}{*}{$\begin{array}{l}\text { Frequência de consumo de frutas e saladas verdes no dia } \\
\text { anterior à coleta de dados }{ }^{22} \text {. }\end{array}$} & Sem risco: $\geq 3$ vezes \\
\hline & & Com risco: $<3$ vezes \\
\hline \multirow{2}{*}{ Consumo de embutidos } & \multirow{2}{*}{$\begin{array}{l}\text { Frequência de consumo de embutidos no dia anterior à coleta } \\
\text { de dados }{ }^{22} \text {. }\end{array}$} & Sem risco: nenhuma vez \\
\hline & & Com risco: $\geq 1 \mathrm{vez}$ \\
\hline \multirow{2}{*}{ Tabagismo diário } & \multirow{2}{*}{$\begin{array}{l}\text { Consumo diário de pelo menos um cigarro, nos } 30 \text { dias } \\
\text { anteriores à coleta de dados. }\end{array}$} & Sem risco: não \\
\hline & & Com risco: sim \\
\hline \multirow{2}{*}{$\begin{array}{l}\text { Consumo abusivo de } \\
\text { bebida alcoólica }\end{array}$} & \multirow{2}{*}{$\begin{array}{l}\text { Consumo de cinco ou mais doses de bebida alcoólica em uma } \\
\text { mesma ocasião, nos } 30 \text { dias anteriores à coleta de dados }{ }^{16,23} \text {. }\end{array}$} & Sem risco: nenhum dia \\
\hline & & Com risco: $\geq 1$ dia \\
\hline \multirow{2}{*}{ Consumo de drogas ilícitas } & \multirow{2}{*}{$\begin{array}{l}\text { Consumo de drogas ilícitas (maconha, cocaína, crack, esteroides } \\
\text { anabólicos, heroína, LSD e êxtase) alguma vez na vida. }\end{array}$} & Sem risco: nenhuma vez \\
\hline & & Com risco: $\geq 1 \mathrm{vez}$ \\
\hline \multirow{2}{*}{ Envolvimento em brigas } & \multirow{2}{*}{$\begin{array}{l}\text { Envolvimento em brigas nos } 12 \text { meses que antecederam a coleta } \\
\text { de dados. }\end{array}$} & Sem risco: nenhuma vez \\
\hline & & Com risco: $\geq 1 \mathrm{vez}$ \\
\hline \multirow{2}{*}{$\begin{array}{l}\text { Uso irregular de } \\
\text { preservativo nas relações } \\
\text { sexuais }\end{array}$} & \multirow{2}{*}{$\begin{array}{l}\text { Frequência de uso de preservativo nas relações sexuais (para } \\
\text { aqueles que têm vida sexual ativa). }\end{array}$} & Sem risco: sempre \\
\hline & & Com risco: raramente/nunca \\
\hline
\end{tabular}


Quadro 1. Continuação...

\begin{tabular}{|c|c|c|}
\hline Variáveis & Medidas (autorreferidas) & Categorias \\
\hline \multicolumn{3}{|c|}{ Características sociodemográficas } \\
\hline \multirow{2}{*}{ Gênero } & \multirow{2}{*}{ Classificação dos acadêmicos quanto ao sexo } & Feminino \\
\hline & & Masculino \\
\hline \multirow{2}{*}{ Faixa etária } & \multirow{2}{*}{ Faixa de idade do acadêmico } & Acima de 21 anos \\
\hline & & Até 21 anos \\
\hline \multirow{2}{*}{ Cor de pele } & \multirow{2}{*}{ Classificação quanto à etnia autodeclarada } & Branca \\
\hline & & Outra: negro/asiático/índio \\
\hline \multirow{2}{*}{ Estado civil } & \multirow{2}{*}{ Estado civil do acadêmico } & $\begin{array}{l}\text { Sem companheiro: solteiro/viúvo/ } \\
\text { divorciado/ separado }\end{array}$ \\
\hline & & $\begin{array}{l}\text { Com companheiro: casado(a)/união } \\
\text { estável }\end{array}$ \\
\hline \multirow{2}{*}{ Trabalho } & \multirow{2}{*}{ Condição trabalhista } & Não Trabalha \\
\hline & & Trabalha \\
\hline \multirow{2}{*}{ Classe econômica } & \multirow{2}{*}{ Segundo Critério de Classificação Econômica Brasil ${ }^{19}$. } & Classe C ou D \\
\hline & & Classe A ou B \\
\hline \multicolumn{3}{|c|}{ Características acadêmicas } \\
\hline \multirow[b]{2}{*}{ Área de graduação } & \multirow{2}{*}{$\begin{array}{l}\text { Área em que estava cursando a graduação no período da coleta } \\
\text { de dados }\end{array}$} & Ciências Biológicas e da Saúde \\
\hline & & $\begin{array}{l}\text { Outras: Humanas, Exatas e Sociais } \\
\text { Aplicadas }\end{array}$ \\
\hline \multirow[t]{2}{*}{ Campus } & Campus em que estava cursando a graduação no & Montes Claros \\
\hline & período da coleta de dados & Outros \\
\hline \multirow{2}{*}{ Turno de estudo } & \multirow{2}{*}{$\begin{array}{l}\text { Turno do dia em que estava matriculado no período da coleta } \\
\text { de dados }\end{array}$} & Diurno \\
\hline & & Noturno \\
\hline \multirow{2}{*}{ Período de estudo } & \multirow{2}{*}{$\begin{array}{l}\text { Período em que estava cursando a graduação durante a coleta } \\
\text { dos dados }\end{array}$} & $1^{\circ}$ ao $4^{\circ}$ período \\
\hline & & $5^{\circ}$ período em diante \\
\hline
\end{tabular}

\section{RESULTADOS}

Participaram deste estudo 902 estudantes, sendo $20,4 \%$ da área de saúde, $16,0 \%$ de exatas, $36,2 \%$ de humanas e $27,4 \%$ sociais. A idade dos estudantes variou de 18 a 45 anos, com média igual a 22 anos, 67,1\% era do gênero feminino e 82,2\% pertencia às classes econômicas C ou D. Os CRS mais prevalentes foram o baixo consumo de frutas e verduras $(98,1 \%)$, não realização de exercício aeróbio $(71,2 \%)$ e uso irregular de preservativo $(63,1 \%)$. A distribuição das demais variáveis analisadas, bem como as prevalências dos CRS e seus intervalos de confiança de $95 \%$, estão apresentados na Tabela 1 .

Os resultados da análise bivariada revelaram maiores prevalências de consumo abusivo de bebidas alcoólicas, consumo de drogas ilícitas e envolvimento em brigas entre os homens. O gênero feminino apresentou maiores prevalências dos seguintes CRS: não realização de exercício aeróbio, baixo consumo de frutas e verduras, consumo de embutidos e uso irregular de preservativo nas relações sexuais (Tabela 2 ).

Quanto à faixa etária, os universitários com até 21 anos consumiram mais embutidos e se envolveram mais em brigas, enquanto aqueles com idade acima de 21 anos usaram preservativo de modo irregular com maior frequência. Por estado civil, os universitários que possuíam companheiro (casamento/união estável) foram os que apresentaram maiores prevalências de não realização de exercício aeróbio e uso irregular de preservativo (Tabela 2).

Com relação à área de graduação, foi verificado entre os acadêmicos de Ciências Biológicas e da Saúde maior consumo de embutidos, bebidas alcoólicas e drogas ilícitas e, entre os estudantes de outras áreas de graduação, maiores prevalências de não realização de exercício aeróbio e uso irregular de preservativo. $\mathrm{O}$ consumo de drogas foi mais prevalente entre os universitários do campus de Montes Claros, e o uso irregular de preservativo entre estudantes de outros campi. Quanto à classe econômica, os estudantes pertencentes às famílias das classes A ou B referiram maior consumo de embutidos, bebidas alcoólicas e drogas ilícitas, comparados àqueles pertencentes às classes $\mathrm{C}$ ou $\mathrm{D}$ (Tabela 2 ).

$\mathrm{Na}$ análise múltipla, após o ajuste para as variáveis de cada modelo, foram identificados os seguintes subgrupos associados aos CRS: (a) gênero masculino (consumo abusivo de bebida alcoólica, consumo de drogas ilícitas e envolvimento em brigas); (b) idade até 21 anos (consumo de embutidos e envolvimento em brigas); (c) estado civil com companheiro (envolvimento em brigas e uso irregular de preservativo); (d) outras áreas de graduação (não realização de exercício aeróbio); (e) classes econômicas A ou B (consumo de embutidos e consumo de drogas ilícitas) (Tabela 3). 


\section{DISCUSSÃO}

O presente estudo estimou a prevalência de CRS em uma população de universitários do norte de Minas Gerais, bem como os fatores associados a esses comportamentos. Constatou-se que os CRS mais prevalentes adotados por esses universitários foram baixo consumo de frutas e verduras, não realização de exercício aeróbio e uso irregular de preservativo, e os com menores prevalências foram tabagismo diário, envolvimentos em brigas e consumo de drogas ilícitas.
Foram constatados hábitos alimentares inadequados, como o baixo consumo de frutas e verduras reportado por quase todos os universitários entrevistados, corroborando os resultados de estudo prévio realizado em Santa Catarina ${ }^{24}$. Verificou-se também expressiva prevalência do consumo de embutidos, sugerindo correlação entre as atividades acadêmicas e a opção por lanches rápidos, pouco nutritivos e de elevado valor calórico ${ }^{24}$.

Maior prevalência de consumo de embutidos foi observada entre universitários com idade até 21 anos e pertencentes às

Tabela 1. Distribuição dos jovens matriculados em cursos de graduação de uma instituição pública no norte de Minas Gerais, segundo comportamentos de risco à saúde, características sociodemográficas e acadêmicas. Montes Claros, Brasil, 2013

\begin{tabular}{|c|c|c|c|c|}
\hline Variável & Categoria & $\mathbf{n}$ & $\%$ & IC(95\%) \\
\hline \multicolumn{5}{|l|}{ Comportamentos de risco } \\
\hline \multirow{2}{*}{ Não realização de exercício aeróbio } & Sem risco: $\geq 3$ dias & 260 & 28,8 & - \\
\hline & Com risco $<3$ dias & 642 & 71,2 & $(68,2 ; 74,2)$ \\
\hline \multirow{2}{*}{ Baixo consumo de frutas e verduras } & Sem risco: $\geq 3$ vezes & 17 & 1,9 & - \\
\hline & Com risco: $<3$ vezes & 885 & 98,1 & $(97,2 ; 98,9)$ \\
\hline \multirow{2}{*}{ Consumo de embutidos } & Sem risco: nenhuma vez & 470 & 52,1 & - \\
\hline & Com risco: $\geq 1 \mathrm{vez}$ & 432 & 47,9 & $(44,6 ; 51,2)$ \\
\hline \multirow{2}{*}{ Tabagismo diário } & Sem risco: não & 887 & 98,3 & - \\
\hline & Com risco: $\operatorname{sim}$ & 15 & 1,7 & $(0,9 ; 2,5)$ \\
\hline \multirow{2}{*}{ Consumo abusivo de bebida alcoólica } & Sem risco: nenhum dia & 639 & 70,8 & - \\
\hline & Com risco: $\geq 1$ dia & 263 & 29,2 & $(26,2 ; 32,2)$ \\
\hline \multirow{2}{*}{ Consumo de drogas ilícitas } & Sem risco: nenhuma vez & 822 & 91,1 & - \\
\hline & Com risco: $\geq 1 \mathrm{vez}$ & 80 & 8,9 & $(7,0 ; 10,8)$ \\
\hline \multirow{2}{*}{ Envolvimento em brigas } & Sem risco: nenhuma vez & 864 & 95,8 & - \\
\hline & Com risco: $\geq 1$ vez & 38 & 4,2 & $(2,9 ; 5,5)$ \\
\hline \multirow{2}{*}{ Uso irregular de preservativo nas relações sexuais } & Sem risco: sempre & 174 & 36,9 & - \\
\hline & Com risco: raramente/nunca & 297 & 63,1 & $(59,9 ; 66,2)$ \\
\hline \multicolumn{5}{|l|}{ Características sociodemográficas } \\
\hline \multirow{2}{*}{ Gênero } & Feminino & 605 & 67,1 & \\
\hline & Masculino & 297 & 32,9 & \\
\hline \multirow{2}{*}{ Faixa etária } & Acima de 21 anos & 395 & 43,8 & \\
\hline & Até 21 anos & 507 & 56,2 & \\
\hline \multirow{2}{*}{ Cor de pele } & Branca & 343 & 38,0 & \\
\hline & Outra & 559 & 62,0 & \\
\hline \multirow{2}{*}{ Estado civil } & Sem companheiro & 781 & 86,6 & \\
\hline & Com companheiro & 121 & 13,4 & \\
\hline \multirow[t]{2}{*}{ Trabalho } & Não trabalha & 375 & 41,6 & \\
\hline & Trabalha & 527 & 58,4 & \\
\hline \multirow[t]{2}{*}{ Classe econômica } & Classe C ou D & 741 & 82,2 & \\
\hline & Classe A ou B & 161 & 17,8 & \\
\hline \multicolumn{5}{|l|}{ Características acadêmicas } \\
\hline & Ciências Biológicas e da Saúde & 184 & 20,4 & \\
\hline & Ciências Humanas & 327 & 36,2 & \\
\hline \multirow[t]{2}{*}{ Área de graduação } & Ciências Exatas e Tecnológicas & 144 & 16,0 & \\
\hline & Ciências Sociais Aplicadas & 247 & 27,4 & \\
\hline \multirow{2}{*}{ Campus } & Montes Claros & 596 & 66,1 & \\
\hline & Outros & 306 & 33,9 & \\
\hline \multirow{2}{*}{ Turno de estudo } & Diurno & 378 & 41,9 & \\
\hline & Noturno & 524 & 58,1 & \\
\hline \multirow{2}{*}{ Período de graduação } & $1^{\circ}$ ao $4^{\circ}$ período & 532 & 59,0 & \\
\hline & $5^{\circ}$ período em diante & 370 & 41,0 & \\
\hline
\end{tabular}


Tabela 2. Prevalência e razão de prevalência bruta (RP) dos comportamentos de risco à saúde em universitários de uma instituição pública no norte de Minas Gerais, Brasil, 2013

\begin{tabular}{|c|c|c|c|c|c|c|c|c|}
\hline Variável & NREA (\%) & BCFV (\%) & CE (\%) & TBD (\%) & CABA (\%) & CDI (\%) & EB (\%) & UIPRS (\%) \\
\hline \multicolumn{9}{|l|}{ Gênero } \\
\hline Feminino $^{*}$ & 73,4 & 98,5 & 48,1 & 1,7 & 24,8 & 6,0 & 3,1 & 65,4 \\
\hline Masculino & 66,7 & 97,3 & 47,5 & 1,7 & 38,0 & 14,8 & 6,4 & 58,8 \\
\hline $\mathrm{RP}$ & 0,91 & 0,99 & 0,99 & 1,02 & 1,54 & 2,49 & 2,04 & 0,90 \\
\hline (IC 95\%) & $(0,83-1,00)$ & $(0,97-1,01)$ & $(0,85-1,14)$ & $(0,35-2,95)$ & $(1,26-1,88)$ & $(1,64-3,78)$ & $(1,10-3,79)$ & $(0,77-1,05)$ \\
\hline \multicolumn{9}{|l|}{ Faixa etária } \\
\hline Acima de 21 anos $^{\star}$ & 72,2 & 98,0 & 41,8 & 2,0 & 29,1 & 8,6 & 2,8 & 71,8 \\
\hline Até 21 anos & 70,4 & 98,2 & 52,7 & 1,4 & 29,2 & 9,1 & 5,3 & 52,4 \\
\hline $\mathrm{RP}$ & 0,98 & 1,00 & 1,26 & 0,68 & 1,00 & 1,05 & 1,91 & 0,73 \\
\hline (IC 95\%) & $(0,90-1,06)$ & $(0,98-1,02)$ & $(1,09-1,45)$ & $(0,25-1,86)$ & $(0,82-1,23)$ & $(0,69-1,61)$ & $(0,96-3,81)$ & $(0,63-0,85)$ \\
\hline \multicolumn{9}{|l|}{ Estado civil } \\
\hline Sem companheiro* & 70,2 & 98,0 & 48,9 & 1,4 & 29,1 & 8,7 & 4,0 & 54,1 \\
\hline Com companheiro & 77,7 & 99,2 & 41,3 & 3,3 & 29,8 & 9,9 & 5,8 & 91,2 \\
\hline $\mathrm{RP}$ & 1,11 & 1,01 & 0,85 & 2,33 & 1,02 & 1,12 & 1,45 & 1,69 \\
\hline (IC 95\%) & $(1,00-1,23)$ & $(0,99-1,03)$ & $(0,68-1,05)$ & $(0,76-7,14)$ & $(0,76-1,37)$ & $(0,64-2,04)$ & $(0,66-3,23)$ & $(1,52-1,89)$ \\
\hline \multicolumn{9}{|l|}{ Área de graduação } \\
\hline Ciências B. Saúde* & 59,8 & 99,5 & 53,3 & 1,6 & 32,1 & 11,4 & 3,3 & 54,6 \\
\hline Outras áreas & 74,1 & 97,8 & 46,5 & 1,7 & 28,4 & 8,2 & 4,5 & 65,2 \\
\hline $\mathrm{RP}$ & 1,24 & 0,98 & 0,87 & 1,03 & 0,89 & 0,72 & 1,37 & 1,19 \\
\hline (IC 95\%) & $(1,09-1,41)$ & $(0,97-1,00)$ & $(0,75-1,02)$ & $(0,29-3,60)$ & $(0,70-1,13)$ & $(0,45-1,15)$ & $(0,58-3,22)$ & $(0,98-1,45)$ \\
\hline \multicolumn{9}{|l|}{ Campus } \\
\hline M. Claros ${ }^{*}$ & 70,8 & 97,8 & 49,2 & 1,3 & 28,0 & 10,4 & 4,7 & 59,6 \\
\hline Outros & 71,9 & 98,7 & 45,4 & 2,3 & 31,4 & 5,9 & 3,3 & 68,0 \\
\hline $\mathrm{RP}$ & 1,02 & 1,01 & 0,92 & 1,70 & 1,12 & 0,57 & 0,70 & 1,14 \\
\hline (IC 95\%) & $(0,93-1,11)$ & $(0,99-1,03)$ & $(0,80-1,07)$ & $(0,62-4,66)$ & $(0,91-1,38)$ & $(0,34-0,94)$ & $(0,34-1,41)$ & $(1,00-1,31)$ \\
\hline \multicolumn{9}{|l|}{ Classe econômica } \\
\hline $\mathrm{C}$ ou $\mathrm{D}^{*}$ & 72,3 & 98,0 & 46,0 & 1,5 & 27,9 & 7,3 & 4,0 & 62,5 \\
\hline A ou B & 65,8 & 98,8 & 56,5 & 2,5 & 34,8 & 16,1 & 5,0 & 65,2 \\
\hline $\mathrm{RP}$ & 0,91 & 1,01 & 1,23 & 1,67 & 1,25 & 2,22 & 1,22 & 1,04 \\
\hline (IC 95\%) & $(0,81-1,02)$ & $(0,99-1,03)$ & $(1,05-1,43)$ & $(0,54-5,26)$ & $(0,98-1,59)$ & $(1,43-3,45)$ & $(0,57-2,63)$ & $(0,88-1,23)$ \\
\hline
\end{tabular}

${ }^{*}$ Categoria de referência. As variáveis cor de pele, turno, período e trabalho apresentaram valor-p >0,20 e não foram inseridas na tabela. NREA: Não realização de exercício aeróbio; BCFV: Baixo consumo de frutas e verduras; CE: Consumo de embutidos; TBD: Tabagismo diário; CABA: Consumo abusivo de bebida alcoólica; CDI: Consumo de drogas ilícitas; EB: Envolvimento em brigas; UIPRS: Uso irregular de preservativo nas relações sexuais

classes A ou B, assim como os achados de outros estudos ${ }^{24,25}$. Observa-se que, com o avançar da idade, ao adquirir experiência e maturidade, os universitários se adaptam mais à rotina de estudos e tendem a melhorar os cuidados com a saúde ${ }^{26}$, inclusive com a alimentação. Os jovens de classe econômica mais elevada possuem melhor condição financeira para alimentar-se fora de casa, e assim estão mais expostos às ofertas de refeições rápidas com alimentos industrializados ${ }^{24,25}$, possivelmente seja essa a explicação da associação observada entre consumo de embutidos e classes econômica A ou B no presente estudo.

O segundo CRS mais prevalente entre os jovens foi a não realização de exercício aeróbio. Esse CRS é comum entre os universitários por motivos de falta de tempo, motivação e apoio social, bem como pela ausência de espaços destinados à realização de exercícios ${ }^{27}$. Embora resultados semelhantes tenham sido encontrados em universitários portugueses ${ }^{4}$, esse é um problema regional que não se repete em vários estudos nacionais e internacionais ${ }^{1,5,27}$. A inatividade física é um comportamento que merece atenção por ter sido referida como um relevante fator de risco para agravos à saúde, especialmente em portadores de doenças crônicas não transmissíveis ${ }^{4,6}$.

A não realização de exercício aeróbio foi mais frequente em estudantes que não eram da área de Ciências Biológicas e da Saúde. Esse achado pode ser reflexo dos conteúdos aprendidos e matérias específicas dos cursos de biológicas e da saúde. É importante salientar que o curso de Educação Física também está incluído na área de Ciências Biológicas e da Saúde, e pode ter interferido nesse resultado. Em um estudo em Recife, PE, estudantes de Educação Física foram os únicos que desempenhavam atividades físicas, enquanto universitários dos outros cursos eram predominantemente sedentários ${ }^{14}$. 
Tabela 3. Razão de prevalência ajustada $(\mathrm{RP})$ para comportamentos de risco à saúde em universitários de uma instituição pública no norte de Minas Gerais, Brasil, 2013

\begin{tabular}{|c|c|c|c|c|c|c|}
\hline Variável & $\begin{array}{c}\text { NREA } \\
\text { RP } \\
\text { (IC95\%) }\end{array}$ & $\begin{array}{c}\text { CE } \\
\text { RP } \\
(\mathrm{IC} 95 \%)\end{array}$ & $\begin{array}{c}\text { CABA } \\
\text { RP } \\
(\mathrm{IC} 95 \%)\end{array}$ & $\begin{array}{c}\text { CDI } \\
\text { RP } \\
(\text { IC95\%) }\end{array}$ & $\begin{array}{c}\text { EB } \\
\text { RP } \\
(\text { IC95\%) }\end{array}$ & $\begin{array}{c}\text { UIPRS } \\
\text { RP } \\
\text { (IC95\%) }\end{array}$ \\
\hline \multicolumn{7}{|l|}{ Gênero } \\
\hline Feminino $^{*}$ & 1,00 & 1,00 & 1,00 & 1,00 & 1,00 & 1,00 \\
\hline Masculino & $\begin{array}{c}0,91 \\
(0,83-1,00)\end{array}$ & $\begin{array}{c}0,98 \\
(0,85-1,14)\end{array}$ & $\begin{array}{c}1,53 \\
(1,25-1,87)\end{array}$ & $\begin{array}{c}2,41 \\
(1,59-3,63)\end{array}$ & $\begin{array}{c}2,12 \\
(1,13-3,99)\end{array}$ & $\begin{array}{c}0,93 \\
(0,80-1,07)\end{array}$ \\
\hline \multicolumn{7}{|l|}{ Faixa etária } \\
\hline Acima de 21 anos$^{*}$ & 1,00 & 1,00 & 1,00 & 1,00 & 1,00 & 1,00 \\
\hline Até 21 anos & $\begin{array}{c}1,02 \\
(0,93-1,12)\end{array}$ & $\begin{array}{c}1,23 \\
(1,05-1,43)\end{array}$ & $\begin{array}{c}1,03 \\
(0,82-1,28)\end{array}$ & $\begin{array}{c}1,10 \\
(0,71-1,71)\end{array}$ & $\begin{array}{c}2,77 \\
(1,23-6,22)\end{array}$ & $\begin{array}{c}0,87 \\
(0,74-1,03)\end{array}$ \\
\hline \multicolumn{7}{|l|}{ Estado civil } \\
\hline Sem companheiro* & 1,00 & 1,00 & 1,00 & 1,00 & 1,00 & 1,00 \\
\hline Com companheiro & $\begin{array}{c}1,10 \\
(0,98-1,25)\end{array}$ & $\begin{array}{c}0,97 \\
(0,76-1,23)\end{array}$ & $\begin{array}{c}1,04 \\
(0,76-1,43)\end{array}$ & $\begin{array}{c}1,64 \\
(0,88-3,03)\end{array}$ & $\begin{array}{c}3,03 \\
(1,19-7,69)\end{array}$ & $\begin{array}{c}1,59 \\
(1,39-1,82)\end{array}$ \\
\hline \multicolumn{7}{|l|}{ Área de graduação } \\
\hline Ciências B. Saúde ${ }^{*}$ & 1,00 & 1,00 & 1,00 & 1,00 & 1,00 & 1,00 \\
\hline Outras & $\begin{array}{c}1,26 \\
(1,11-1,43)\end{array}$ & $\begin{array}{c}0,92 \\
(0,78-1,08)\end{array}$ & $\begin{array}{c}0,84 \\
(0,66-1,07)\end{array}$ & $\begin{array}{c}0,79 \\
(0,49-1,30)\end{array}$ & $\begin{array}{c}1,62 \\
(0,70-3,78)\end{array}$ & $\begin{array}{c}1,09 \\
(0,90-1,33)\end{array}$ \\
\hline \multicolumn{7}{|l|}{ Classe econômica } \\
\hline $\mathrm{C}$ ou $\mathrm{D}^{*}$ & 1,00 & 1,00 & 1,00 & 1,00 & 1,00 & 1,00 \\
\hline A ou B & $\begin{array}{c}0,92 \\
(0,81-1,03)\end{array}$ & $\begin{array}{c}1,20 \\
(1,03-1,41)\end{array}$ & $\begin{array}{c}1,22 \\
(0,96-1,56)\end{array}$ & $\begin{array}{c}1,92 \\
(1,25-2,94)\end{array}$ & $\begin{array}{c}1,12 \\
(0,52-2,44)\end{array}$ & $\begin{array}{c}1,16 \\
(0,98-1,39)\end{array}$ \\
\hline Deviance & 429,354 & 627,357 & 632,631 & 357,669 & 225,963 & 253,791 \\
\hline p-valor: & 0,480 & 0,701 & 0,707 & 0,400 & 0,252 & 0,547 \\
\hline
\end{tabular}

${ }^{*}$ Categoria de referência. NREA: Não realização de exercício aeróbio; CE: Consumo de embutidos; CABA: Consumo abusivo de bebida alcoólica; CDI: Consumo de drogas ilícitas; EB: Envolvimento em brigas; UIPRS: Uso irregular de preservativo nas relações sexuais

O terceiro CRS, reportado por mais da metade da amostra estudada, foi o uso irregular de preservativo nas relações sexuais. Semelhante resultado foi observado entre universitários pernambucanos ${ }^{28}$, e valores ainda superiores foram descritos em outro estudo com universitários da área da Saúde em Minas Gerais $^{29}$. Pela escolaridade da população-alvo, não é possível atribuir o uso irregular de preservativo à falta de informação. Esse hábito depende também do significado da relação afetiva que o jovem tem com seu parceiro, do envolvimento emocional no momento da relação sexual e do acesso aos métodos contraceptivos ${ }^{29}$. O uso irregular de preservativo foi mais prevalente entre aqueles que viviam com companheiro. Essa associação já era esperada, visto que a estabilidade do relacionamento gera confiança entre os parceiros, levando ao uso irregular de preservativo nas relações sexuais ${ }^{30}$.

O consumo abusivo de bebida alcoólica foi relatado por cerca de um terço da população estudada. Estudos apontam que o álcool é a droga mais consumida por universitários e pode estar associado a outros CRS, como direção sob o efeito de bebidas, consumo de tabaco e de drogas ilícitas ${ }^{11,31}$, relação sexual desprotegida ${ }^{11,32}$, envolvimento em brigas e ocasiões de violência ${ }^{5}$. As bebidas alcoólicas são usadas como estratégia de descontração, alívio de tensão e fortalecimento de elos de socialização entre os universitários ${ }^{33}$. Seu consumo é incentivado nas festas universitárias ${ }^{31}$ pelos colegas e mídia ${ }^{32}$. Porém, o consumo habitual e abusivo pode trazer prejuízos para os projetos de vida, para a saúde e para a vida familiar, financeira e social ${ }^{11}$.

Universitários do gênero masculino apresentaram maior prevalência de consumo abusivo de álcool, e também do uso de drogas ilícitas e envolvimento em brigas, corroborando outros estudos $^{5,27,28,31,33,34}$. Jovens do gênero masculino costumam ser mais propensos a exibir CRS por causa de influência social e de fatores culturais de demonstração de poder ${ }^{31,35}$. Porém, o envolvimento em brigas em universitários de Brasília foi mais prevalente entre as mulheres ${ }^{15}$.

O uso de drogas pode ser visto como um comportamento desafiador das regras sociais ${ }^{35}$. Assim como o álcool, as drogas podem expor os usuários a outros CRS, mesmo que seu uso seja esporádico e associado a contextos favoráveis como festas. A prevalência de uso de drogas ilícitas detectada no presente estudo foi inferior à verificada em universitários do Alto Paranaíba, MG $(22,8 \%)^{35}$ e superior ao registrado em estudantes de Porto Velho, RO $(5,3 \%)^{31}$. 
A maior frequência de uso de drogas foi associada a universitários das classes A ou B. Isso ocorre possivelmente devido à acessibilidade financeira e influência do meio social ${ }^{32,36}$. Estudo prévio comprova que a maior renda dos pais está associada com maiores taxas de consumo de maconha e outras drogas ${ }^{37}$.

Apesar dos relatos sobre consumo de drogas e álcool, menos de $2 \%$ dos estudantes declararam fumar diariamente pelo menos um cigarro. Esse resultado foi semelhante ao observado entre universitários no Nordeste do Brasil $(3,1 \%)^{28}$ e em Brasília, DF $(2,5 \%)^{4}$, mas inferior ao encontrado em universitários do Alto Paranaíba, MG $(11,4 \%)^{11}$ e de Porto Velho, RO $(17,5 \%)^{31}$. Divergências entre prevalências podem ocorrer devido às características sociodemográficas e culturais específicas de cada população investigada ${ }^{27}$, mas é possível que a baixa prevalência observada neste estudo esteja relacionada ao sucesso das campanhas e às ações de combate ao tabagismo no Brasil.

$\mathrm{O}$ envolvimento em brigas foi o CRS menos prevalente e mostrou-se associado às variáveis: gênero masculino, idade até 21 anos e estado civil com companheiro. A participação de adolescentes e adultos jovens em brigas pode estar relacionada à imaturidade, à necessidade de autoafirmação e de aprovação pelo grupo de amigos ${ }^{5,38}$, bem como ao uso abusivo de bebidas alcóolicas ${ }^{5}$. Porém, a associação positiva entre o envolvimento em brigas e estado civil com um companheiro é contrária àquela observada em estudos prévios ${ }^{38,39}$. Geralmente, os indivíduos que possuem um companheiro tendem a uma condição afetiva mais estável, com hábitos e comportamentos mais sensatos ${ }^{40}$.

É importante ressaltar que, apesar de a amostra ter sido de uma única universidade, os resultados são representativos de CRS entre estudantes universitários no norte de Minas. Com treze campi, a Unimontes é a maior universidade pública da região e seu corpo discente é composto por alunos do estado de Minas e do sul da Bahia, além de alguns representantes de outras regiões do país. Assim, considera-se que a amostra expressa bem o contexto regional, proporcionando amplo entendimento quanto aos CRS na população de estudo.

\section{CONCLUSÃO}

O estudo demonstrou que os universitários adotaram comportamentos que colocaram a saúde em risco. Os CRS mais prevalentes foram o baixo consumo de frutas e verduras, a não realização de exercício aeróbio e o uso irregular de preservativo nas relações sexuais. Destaca-se que o consumo de álcool, drogas ilícitas e envolvimento em brigas foram os CRS mais prevalentes entre os estudantes do sexo masculino.

Os CRS mostraram-se positivamente associados ao gênero masculino (consumo abusivo de bebida alcoólica, consumo de drogas ilícitas e envolvimento em brigas), idade até 21 anos (consumo de embutidos e envolvimento em brigas), estado civil com companheiro (envolvimento em brigas e uso irregular de preservativo nas relações sexuais), estudantes de outras áreas de graduação (não realização de exercício aeróbio) e classe econômica A ou B (consumo de embutidos e consumo de drogas ilícitas).

Os resultados encontrados sugerem que novos modos de comportamentos de saúde devam ser adotados pela população universitária do norte de Minas, MG. Recomenda-se que as instituições de ensino superior realizem mais pesquisas, inclusive prospectivas, com população universitária para identificar e compreender melhor os CRS no espaço de tempo do período acadêmico.

\section{REFERÊNCIAS}

1. Eaton DK, Kann L, Kinchen S, Shanklin S, Flint KH, Hawkins J, et al. Youth risk behavior surveillance - United States, 2011. MMWR Surveill Summ. 2012;61(4):1-162. PMid:22673000.

2. El Ansari W, Stock C, John J, Deeny P, Phillips C, Snelgrove S, et al. Health promoting behaviours and lifestyle characteristics of students at seven universities in the UK. Cent Eur J Public Health. 2011;19(4):197-204. PMid:22432394.

3. Varela-Mato V, Cancela JM, Ayan C, Martin V, Molina A. Lifestyle and health among Spanish university students: differences by gender and academic discipline. Int J Environ Res Public Health. 2012;9(12):2728-41. PMid:23066393. http://dx.doi.org/10.3390/ijerph9082728.

4. Brandão MP, Pimentel FL, Cardoso MF. Impact of academic exposure on health status of university students. Rev Saúde Pública. 2011;45(1):49-58. PMid:21203695. http://dx.doi.org/10.1590/S0034-89102011000100006.

5. Farias Jr JC, Nahas MV, Barros MV, Loch MR, Oliveira ES, De Bem MF, et al Comportamentos de risco à saúde em adolescentes no Sul do Brasil: prevalência e fatores associados. Rev Panam Salud Publica. 2009;25(4):34452. PMid:19531323. http://dx.doi.org/10.1590/S1020-49892009000400009.

6. Legnani E, Legnani RF, Dellagrana RA, Silva MP, Barbosa Fo VC, Campos W. Comportamentos de risco à saúde e excesso de peso corporal em escolares de Toledo, Paraná, Brasil. Motri. 2012;8(3):59-70.

7. Malta DC, Sardinha LMV, Mendes I, Barreto SM, Giatti L, Castro IRR, et al. Prevalência de fatores de risco e proteção de doenças crônicas não transmissíveis em adolescentes: resultados da Pesquisa Nacional de Saúde do Escolar (PeNSE), Brasil, 2009. Ciênc. Saúde Colet. 2010;15(Supl 2):3009-19. http://dx.doi.org/10.1590/S1413-81232010000800002.

8. Heckmann W, Silveira CM. Dependência doálcool: aspectos clínicos e diagnósticos. In: Andrade AG, Anthony JC, Silveira CM. Álcool e suas consequências: uma abordagem multiconceitual. Barueri (SP): Minha Editora; 2009. p. 67-87.

9. Feijão EP, Sampaio HAC, Sabry MOD, Carioca AAFC, Yum MEM, Lima JWO. Prática de binge alcoólico entre estudantes universitários. Rev. Bras. Promoç. Saúde. 2010;25(4):462-8.

10. Giacomozzi AI, Itokasu MC, Luzardo AR, Figueiredo CDS, Vieira M. Levantamento sobre uso de álcool e outras drogas e vulnerabilidades relacionadas de estudantes de escolas públicas participantes do programa saúde do escolar/ saúde e prevenção nas escolas no município de Florianópolis. Saúde Soc. 2012;21(3):612-22. http://dx.doi.org/10.1590/S0104-12902012000300008. 
11. Antoniassi Jr G, Meneses Gaya C. Uso de droga associado ao comportamento de risco universitário. Saúde e Pesqui. 2015;8:9-17.

12. Matsudo SM, Matsudo VR, Araújo T, Andrade D, Andrade E, Oliveira L, et al. Nivel de AF da população do Estado de São Paulo: análise de acordo com o gênero, idade, nível socioeconômico, distribuição geográfica e de conhecimento. Rev. Bras. Cien. e Mov. 2002;10(4):41-50.

13. Instituto Brasileiro de Geografia e Estatística. Pesquisa Nacional de Saúde 2013: percepção do estado de saúde, estilo de vida e doenças crônicas: Brasil, grandes regiões e unidades da federação [Internet]. Rio de Janeiro: IBGE, Coordenação de Trabalho e Rendimento; 2014 [citado em 2016 nov. 10]. Disponível em: ftp://ftp.ibge.gov.br/PNS/2013/pns2013.pdf

14. Paixão LA, Dias RMR, Prado WL. Estilo de vida e estado nutricional de universitários ingressantes em cursos da área de saúde do Recife, PE. Rev. Bras. Ativ. Fís. Saúde. 2010;15(3):145-50.

15. Faria YO, Gandolfi L, Moura LBA. Prevalência de comportamentos de risco em adulto jovem e universitário. Acta Paul Enferm. 2014;27(6):591-5. http://dx.doi.org/10.1590/1982-0194201400096.

16. Nunes JM, Campolina LR, Vieira MA, Caldeira AP. Consumo de bebidas alcoólicas e prática do binge drinking entre acadêmicos da área da saúde. Rev Psiquiatr Clin. 2012;39(3):94-9. http://dx.doi.org/10.1590/S010160832012000300005

17. Rocha LA, Lopes ACFMM, Martelli DRB, Lima VB, Martelli-Júnior H. Consumo de álcool entre estudantes de faculdades de Medicina de Minas Gerais, Brasil. Rev Bras Educ Med. 2011;35(3):369-75. http://dx.doi. org/10.1590/S0100-55022011000300010.

18. Gasparotto GS. Associação entre o nível de atividade física e fatores de risco cardiovascular em universitários da UFP [dissertação]. Curitiba: Universidade Federal do Paraná; 2012.

19. Associação Brasileira de Empresas de Pesquisa. Critério de Classificação Econômica Brasil: dados com base no levantamento sócio econômico 2000, IBOPE [Internet]. São Paulo: ABEP: 2013 [citado em 2015 mar. 13]. Disponível em: http://www.abep.org/codigosguias/ABEP_CCEB.pdf

20. Bernadelli Jr R. Comportamentos de risco para a saúde de estudantes da Universidade Estadual do Norte do Paraná, Brasil [tese]. Vila Real: Utad; 2010.

21. Lima DF, Levy RB, Luiz OC. Recomendações para atividade física e saúde: consensos, controvérsias e ambiguidades. Rev Panam Salud Publica. 2014;36(3):164-70. PMid:25418766.

22. Brasil. Ministério da Saúde. Secretaria de Atenção à Saúde, Departamento de Atenção Básica. Guia alimentar para a população brasileira [Internet]. 2. ed. Brasília; 2014 [citado em 2015 mar. 13]. Disponível em: http://189.28.128.100/ dab/docs/portaldab/publicacoes/guia_alimentar_populacao_brasileira.pdf

23. National Institute on Alcohol and Alcoholism. Helping patients who drink too much: a clinician's guide. United States: NIAAA; 2015. 40 p. (NIH Publication, 07-3769) [citado em 2015 mar. 13]. Disponível em: http:// pubs.niaaa.nih.gov/publications/Practitioner/CliniciansGuide2005/guide. pdf

24. Teo CRPA, Sá CA, Dall'Agnol P, Welter S. Ambiente alimentar e vulnerabilidade de adolescentes universitários: um estudo com foco no convívio familiar. Rev. Bras. Pesq. Saúde. 2014;16(1):49-58.

25. Lenz A, Olinto MTA, Dias-da-Costa JS, Alves AL, Balbinotti M, Pattussi MP, et al. Socioeconomic, demographic and lifestyle factors associated with dietary patterns of women living in Southern Brazil. Cad Saúde Pública. 2009;25(6):1297-306. PMid:19503960. http://dx.doi.org/10.1590/S0102$311 \times 2009000600012$
26. Jaime PC, Figueiredo ICR, Moura EC, Malta DC. Fatores associados ao consumo de frutas e hortaliças no Brasil, 2006. Rev Saúde Publica. 2009;43(Supl 2):57-64. PMid:19936499. http://dx.doi.org/10.1590/S003489102009000900008 .

27. Sousa TF, José HPM, Barbosa AR. Condutas negativas à saúde em estudantes universitários brasileiros. Ciênc. Saúde Colet. 2013;18(12):3563-75. http:// dx.doi.org/10.1590/S1413-81232013001200013.

28. Colares V, Franca C, Gonzalez E. Condutas de saúde entre universitários: diferenças entre gêneros. Cad Saúde Pública. 2009;25(3):521-8. PMid:19300841. http://dx.doi.org/10.1590/S0102-311X2009000300007.

29. Borges MR, Silveira RE, Santos AS, Lippi UG. Comportamento sexual de ingressantes universitários. R. Pesq.: Cuid. fundam. Online. 2015;7(2):250515

30. Gomes A, Nunes C. Representação social do sexo nos jovens adultos portugueses. Psicol. Reflex. Crit. 2015;28(1):177-85

31. Elicker E, Palazzo LS, Aerts DRGC, Alves GG, Câmara S. Uso de álcool, tabaco e outras drogas por adolescentes escolares de Porto Velho-RO, Brasil. Epidemiol. Serv. Saúde. 2015;24(3):399-410.

32. Pedrosa AAS, Camacho LAB, Passos SRL, Oliveira RVC. Consumo de álcool entre estudantes universitários. Cad Saúde Pública. 2011;27(8):1611-21. PMid:21877009. http://dx.doi.org/10.1590/S0102-311X2011000800016.

33. Rosa LFA, Nascimento ARA. Representações sociais de bebida alcoólica para homens universitários. Arq Bras Psicol. 2015;67(1):3-19.

34. Seguel PF, Santander MG, Ramos SML. Consumo de drogas y factores demográficos associados em estudiantes de primer año de una universidad Chilena. Acta Paul Enferm. 2012;25(2):33-9.

35. Antoniassi Jr G, Meneses Gaya C. Implicações do uso de álcool, tabaco e outras drogas na vida do universitário. Rev Bras Promoção Saúde. 2015b;28(1):67-74. http://dx.doi.org/10.5020/18061230.2015.p67.

36. Malta DC, Mascarenhas MDMM, Porto DL, Barreto SM, Morais No OL. Exposição ao álcool entre escolares e fatores associados. Rev Saúde Pública. 2014;48(1):52-62. PMid:24789637. http://dx.doi.org/10.1590/ S0034-8910.2014048004563.

37. Humensky JL. Are adolescents with high socioeconomic status more likely to engage in alcohol and illicit drug use in early adulthood? Subst Abuse Treat Prev Policy. 2010;5(19):1-10. PMid:20687935.

38. Reis DC, Almeida TAC, Miranda MM, Alves RH, Madeira AMF Vulnerabilidades à saúde na adolescência: condições socioeconômicas, redes sociais, drogas e violência. Rev Lat Am Enfermagem. 2013;21(2):586-94. http://dx.doi.org/10.1590/S0104-11692013000200016.

39. Castro ML, Cunha SS, Souza DPO. Comportamento de violência e fatores associados entre estudantes de Barra do Garças, MT. Rev Saúde Pública. 2011;45(6):1054-61. PMid:21953077. http://dx.doi.org/10.1590/S003489102011005000072 .

40. Loch MR, Bortoletto MSS, Souza RKT, Mesas AE. Simultaneidade de comportamentos de risco para a saúde e fatores associados em estudo de base populacional. Cad Saúde Colet. 2015;23(2):180-7. http://dx.doi. org/10.1590/1414-462X201500020045. 